\title{
Imaging Graphene Field-Effect Transistors on Diamond Using Nitrogen-Vacancy Microscopy
}

\author{
Scott E. Lillie, ${ }^{1,2}$ Nikolai Dontschuk $\odot,{ }^{1,2,}{ }^{*}$ David A. Broadway, ${ }^{1,2}$ Daniel L. Creedon, ${ }^{2}$ \\ Lloyd C.L. Hollenberg, ${ }^{1,2}$ and Jean-Philippe Tetienne ${ }^{2}$ \\ ${ }^{1}$ Centre for Quantum Computation and Communication Technology, School of Physics, The University of \\ Melbourne, Melbourne, Victoria 3010, Australia \\ ${ }^{2}$ School of Physics, The University of Melbourne, Melbourne, Victoria 3010, Australia
}

(Received 30 May 2019; published 9 August 2019)

\begin{abstract}
The application of imaging techniques based on ensembles of nitrogen-vacancy (N- $V$ ) sensors in diamond to characterize electrical devices has been proposed, but the compatibility of $\mathrm{N}-V$ sensing with operational gated devices remains largely unexplored. Here we report the fabrication of graphene fieldeffect transistors directly on the diamond surface and their characterization by $\mathrm{N}-V$ microscopy. The current density within the gated graphene is reconstructed from $\mathrm{N}-V$-magnetometry measurements under both mostly $p$ - and $n$-type doping, but the exact doping level is found to be affected by the measurements. Additionally, we observe a surprisingly large modulation of the electric field at the diamond surface under an applied gate potential, seen in $\mathrm{N}-V$-photoluminescence and $\mathrm{N}$ - $V$-electrometry measurements, suggesting a complex electrostatic response of the oxide-graphene-diamond structure. Possible solutions to mitigate these effects are discussed.
\end{abstract}

DOI: 10.1103/PhysRevApplied.12.024018

\section{INTRODUCTION}

Sensing techniques using nitrogen-vacancy (N- $V$ ) centers in diamond [1] provide a convenient platform by which condensed-matter systems can be interrogated [2]. The local sensitivity of $\mathrm{N}-V$ centers to both magnetic and electric fields, in concert with their flexible experimental conditions [3], permit both sensing and imaging of a range of nanoscale to mesoscale phenomena. An application of these techniques is the characterization of electrical devices and related materials. Magnetic noise spectroscopy has been used to probe and map the local conductivity of thin metallic films [4,5], providing insight into the motion of carriers within the material, and also to identify noise sources from sparse metallic depositions [6]. In lowdimensional systems, magnetic noise spectroscopy should provide insight into local electronic correlations [7,8], whereas static-magnetic-field mapping has been used to map charge transport in carbon nanotubes by scanning single-N- $V$ centers [9], and monolayer graphene ribbons via wide-field imaging experiments [10]. Extensions to the latter scenario should be able to measure signatures of viscous electron flow in graphene and similar systems [11-13] and other mesoscopic effects such as electron-hole puddling [14], and gate-controlled steering of carriers [15].

\footnotetext{
*dontschuk.n@unimelb.edu.au
}

Wide-field imaging of electrical devices using $\mathrm{N}-\mathrm{V}$ ensembles generally requires the devices to be fabricated directly on the $\mathrm{N}-V$-diamond substrate, while many interesting transport phenomena require precise control over the doping in the conductive channel. This can be achieved in a field-effect transistor (FET), using a top gate $[13,16,17]$, an in-plane gate $[18,19]$, or an electrolytic gate [20]. Recently, graphene-based devices have been fabricated successfully on $\mathrm{N}-V$-diamond substrates $[10,13,17]$, but the compatibility of such structures with wide-field $\mathrm{N}-V$ microscopy remains largely unexplored, with questions of whether the operating conditions required for $\mathrm{N}-V$ microscopy may affect the operation and integrity of the FET, or whether the fabrication and operation of the FET may affect the ability to perform $\mathrm{N}-V$ sensing.

In this work, we fabricate a number of top-gated graphene field-effect transistors (GFETs) on a N-Vdiamond substrate, and characterize device phenomena by wide-field imaging of the near-surface $\mathrm{N}-V$ ensemble. Current is injected into the graphene ribbons, $I_{S D}$, by application of a source-drain potential, $V_{S D}$, and the doping of the ribbon is tunable via a top gate potential, $V_{G}$, allowing charge transport to be probed in different doping regimes and for the effect of the gate to be studied [Fig. 1(a)]. Firstly, the devices are characterized by electrical measurements, and the influence of the laser used to excite the N- $V$ layer is assessed. Secondly, the current 
density within the graphene ribbon is reconstructed under $p$ - and $n$-type doping by our measuring the associated Ørsted field by optical readout of the N- $V$ electron-spin resonances. Thirdly, an effect is observed by which the $\mathrm{N}$ - $V$-layer photoluminescence (PL) is modulated by the applied gate potential in regions proximal to the gated device, but extending up to $20 \mu \mathrm{m}$ from the graphene ribbon. Direct measurement of the electric field by the $\mathrm{N}-V$ ensemble electron-spin resonances demonstrate that this effect is due to an enhanced electric field surrounding the graphene ribbon that diminishes the $\mathrm{N}-V^{-}-$to- $\mathrm{N}-V^{0}$ charge-state ratio. Finally, we discuss possible solutions to overcome the challenges identified in this study to facilitate further investigation of transport phenomena in graphene and other two-dimensional materials.

\section{FABRICATION}

GFETs are fabricated on $\mathrm{N}-V$-diamond substrates by use of a standard wet chemical method [21] to transfer monolayer graphene to the substrate from commercially available chemical-vapor-deposition polycrystalline graphene on $\mathrm{Cu}$ foil. The transferred graphene is selectively etched into 20 - or $50-\mu \mathrm{m}$-wide ribbons in an oxygen plasma with a photolithographic resist mask. Multiple photolithography steps are used to create the $\operatorname{Cr}(5 \mathrm{~nm}) / \operatorname{Au}(70 \mathrm{~nm})$ source and drain contacts, wire bonding pads, and the top-gate contact [Fig. 1(b)]. Atomic layer deposition with modified precursor pulses is used to grow an $80-\mathrm{nm} \mathrm{Al}_{2} \mathrm{O}_{3}$ dielectric layer directly on the graphene ribbons without the use of a nucleation layer [22] (Appendix B). The diamond substrates used in these experiments feature a N- $V$ layer formed by ion implantation 10-20 nm below the surface (Appendix A).

\section{GATING EFFECT AND LASER}

After fabrication, the devices are imaged via the $\mathrm{N}-\mathrm{V}$ layer PL with use of a 532-nm continuous-wave (cw) laser to excite the $\mathrm{N}-V$ ensemble and a scientific CMOS camera to collect the PL, filtered around the $\mathrm{N}-V^{-}$phonon side band at $690 \mathrm{~nm}$ [Fig. 1(c)]. Direct visualization of the graphene is made possible by a Förster-resonanceenergy-transfer (FRET) interaction between the graphene ribbon and the near-surface $\mathrm{N}-V$ layer, which quenches the PL [23]. The top gate appears brighter due to enhanced illumination at the $\mathrm{N}-V$ layer under the gate, due to a standing wave formed with the reflected light [24].

Electrical characterization of the devices finds sourcedrain resistances ranging between 7 and $12 \mathrm{k} \Omega$ for the $50-\mu \mathrm{m}$-wide devices $(100 \mu \mathrm{m}$ between source and drain contacts) and between 16 and $91 \mathrm{k} \Omega$ for the $20-\mu \mathrm{m}$ wide devices (400 $\mu \mathrm{m}$ between source and drain contacts), and good Ohmic behavior (Appendix C). Applying a small source-drain potential, $V_{S D}=100 \mathrm{mV}$, and measuring the source-drain current, $I_{S D}$, while sweeping the
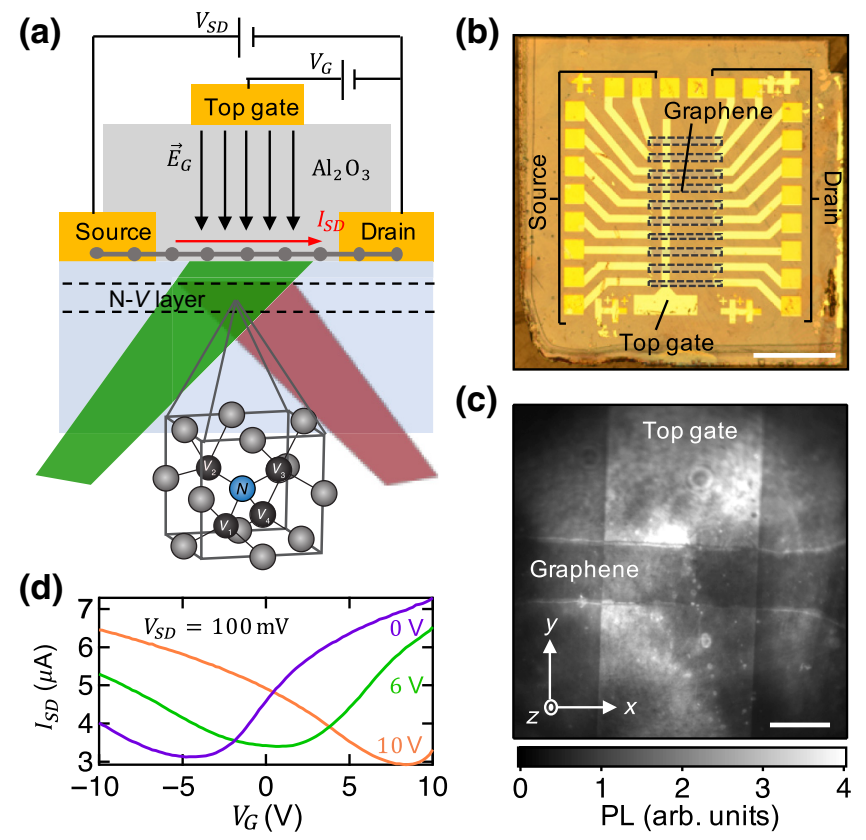

FIG. 1. Imaging GFETs with N-V diamond: (a) Top-gated GFET) fabricated on a $\mathrm{N}-V$-diamond substrate. Current is injected into the graphene ribbon, $I_{S D}$, by application of a sourcedrain potential, $V_{S D}$, while the graphene is doped electrically by application of a top-gate potential, $V_{G}$, which is referenced to the drain. An ensemble of N- $V$ centers is formed $10-20 \mathrm{~nm}$ below the diamond surface, and they exist along all four possible crystallographic orientations, represented by the four possible vacancy positions $\left(V_{1}, V_{2}, V_{3}, V_{4}\right)$ relative to a given $\mathrm{N}$. Readout of the $\mathrm{N}-V$ layer is achieved optically, with use of a 532-nm laser for excitation (green), and the $\mathrm{N}-V$ photoluminescence (red) is collected by a scientific CMOS camera. (b) Photograph of a series of devices fabricated on a $\mathrm{N}$ - $V$-diamond substrate. The scale bar is $500 \mu \mathrm{m}$. (c) PL image of a single GFET. The coordinate system is defined and the scale bar is $20 \mu \mathrm{m}$. (d) $I_{S D^{-}}$ versus- $V_{G}$ curves for a single device measured in the dark after being left to equilibrate at $V_{G}=0,6$, and $10 \mathrm{~V}$ for the purple, green, and orange curves, respectively, under cw-laser illumination, which shifts the conductivity minimum via a photodoping effect (Appendix C).

gate potential, $V_{G}$, we find a conductivity minimum for most devices (Appendix C). The conductivity minima, which indicate the charge-neutrality point, are found to shift significantly depending on the measurement conditions and the history of the device; specifically, the applied gate potential and illumination conditions before the measurement [Fig. 1(d)]. We attribute this to a photonassisted charge transfer between the graphene and the oxide (Appendix C), similar to the optical doping seen with other gate dielectrics and substrates [25-27]. In addition to the photodoping effect, we observe hysteresis in our $I_{S D}$-versus- $V_{G}$ measurements, even when measuring in the dark (Appendix C). The hysteresis is likely due to screening of the electric field associated with the gate 
potential by an accumulation of trapped charge both at the graphene-oxide interface and within the oxide bulk. This has been demonstrated to cause similar hysteresis in graphene devices on $\mathrm{SiO}_{2}$ [28,29], and may also be associated with the defect density within the graphene itself [30]. The presence of the laser is likely to exacerbate this situation by creating additional trapped mobile charges within the oxide [31]. The photodoping induced by the laser is reproducible and sufficiently stable under fixed illumination and gate potentials to maintain the selected majoritycarrier type (Appendix C) over time frames compatible with current-density mapping.

\section{TRANSPORT MAPPING UNDER DOPING}

Previously, N- $V$-ensemble measurements have been used to reconstruct current densities within graphene ribbons from the associated Ørsted field as measured by optically detected magnetic resonance (ODMR) [10]. Here we apply the same method, with the aim of producing current-density maps in the $p$-type-doped and $n$-typedoped regimes. The ODMR measurement is performed with a background magnetic field oriented such that the two electron-spin-resonance transitions of each of the four $\mathrm{N}-V$ orientations are individually resolvable [Fig. 2(a)].
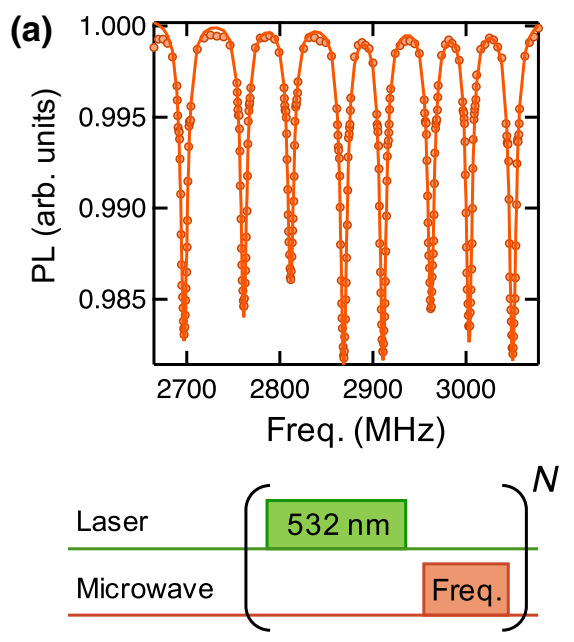

(d)

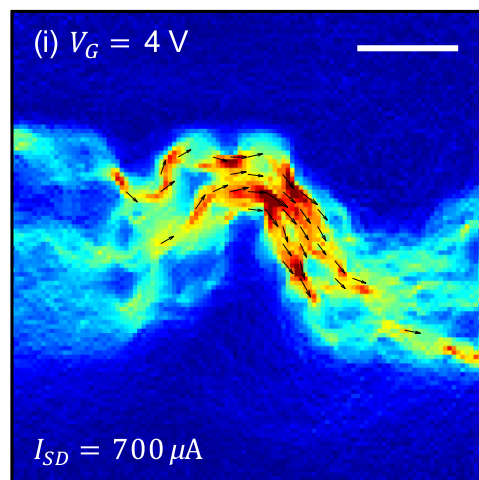

(b)
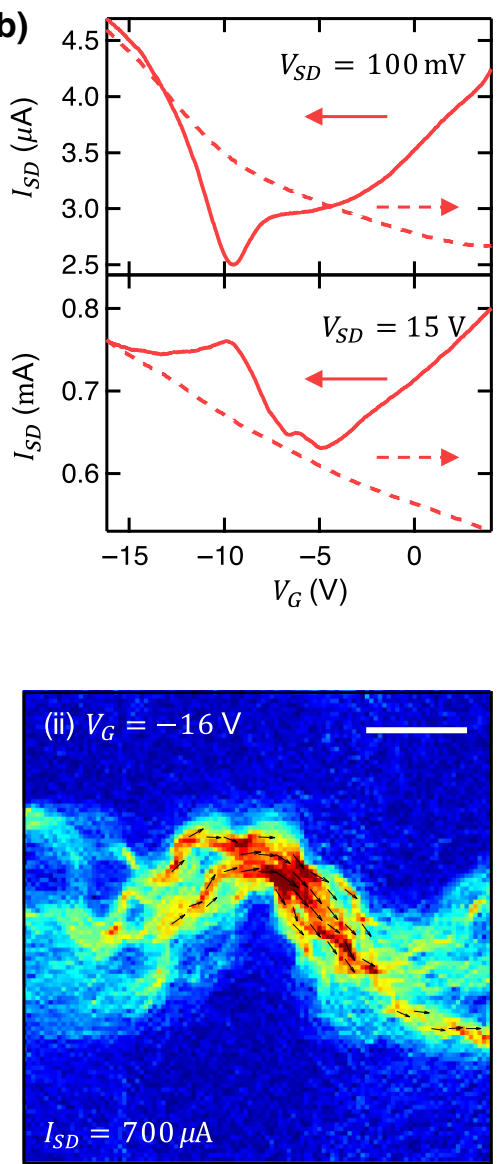

50 (c)

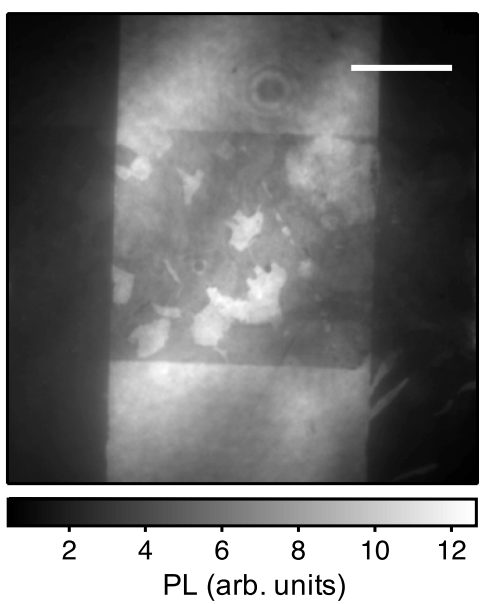

(e)

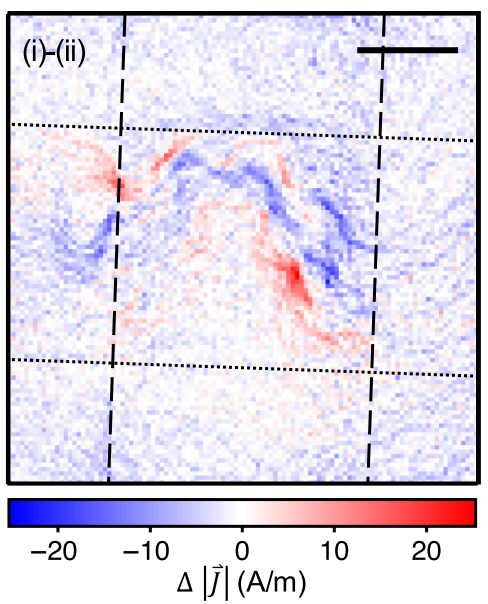

FIG. 2. Current-density mapping for different doping regimes. (a) ODMR spectrum integrated across the field of view of a single GFET (top). The ODMR pulse sequence comprises a single laser pulse to initialize and read out the N- $V$ layer and a microwave pulse to drive $\mathrm{N}-V$ spin transitions resonant with the driving frequency (bottom). A scientic CMOS camera acquires florescence over many repetitions of the pulse sequence for a given microwave frequency. (b) $I_{S D}$ versus $V_{G}$ for the graphene device before current-density mapping at low $V_{S D}$ (top) and high $V_{S D}$ (bottom), representative of that used during acquisition to give $I_{S D}=700 \mu \mathrm{A}$. The device current is left to equilibrate at the initial point of each sweep under laser illumination before the gate potential is swept. Arrows denote the sweep direction. (c) PL image of the GFET to be mapped, showing a number of tears in the graphene due to the transfer process. (d) Vector current-density maps reconstructed from the Ørsted field as measured by ODMR for the GFET under $n$-type doping, (i) $V_{G}=4 \mathrm{~V}$, and $p$-type doping, (ii) $V_{G}=-16 \mathrm{~V}$, each with a constant injected direct current, $I_{S D}=700 \mu \mathrm{A}$. Vector arrows are shown for pixels with a current density greater than $30 \mathrm{~A} / \mathrm{m}$. (e) Difference-in-current-density-magnitude map between the current densities at (i) $V_{G}=4 \mathrm{~V}$ and (ii) $V_{G}=-16 \mathrm{~V}$. The dashed (dotted) lines show the edges of the gate (graphene). All scale bars are $20 \mu \mathrm{m}$. 
Each transition frequency is identified by a reduction in $\mathrm{PL}$ as the $\mathrm{N}-V$ spin-state is driven from the pumped bright state, $|0\rangle$, to the less-fluorescent states, $| \pm 1\rangle$. The magnetic field vector at each pixel is determined by fitting the electron-spin-resonance frequencies, which are shifted by the graphene Ørsted field via the Zeeman effect, and determining the corresponding field projection along each of the four $\mathrm{N}-V$-family axes. Subtracting the background field due to the biasing permanent magnet, the measured Ørsted field is extracted, from which the current density within the graphene ribbon is reconstructed by inverting the BiotSavart law in Fourier space (Appendix D). We note that in these devices, unlike in Ref. [24], no apparent current leakage into the diamond was observed (Appendix D).

Before current-density mapping, the device is characterized electrically to identify $n$ - and $p$-type doping regimes under the conditions used for current-density mapping, which require a comparatively high source-drain potential. $I_{S D}$ is measured over a $V_{G}$ range of -16 to $4 \mathrm{~V}$, with $V_{S D}=100 \mathrm{mV}$ [Fig. 2(b), top] and $V_{S D}=15 \mathrm{~V}$ [Fig. 2(b), bottom], both under cw-laser illumination. Before each $V_{G}$ sweep, the device resistance is left to equilibrate at the initial gate potential under laser illumination, such that the sweep is representative of the device after photodoping and interfacial charge accumulation. For the decreasinggate-potential sweeps (solid curves), conductivity minima are observed at $V_{G}=-9 \mathrm{~V}$ for the smaller $V_{S D}$, and $V_{G}=-5 \mathrm{~V}$ for the larger $V_{S D}$, which also shows a different shape in the transport curve. This is likely due to $V_{G}$ being referenced to the drain contact, and $V_{G}$ being of comparable magnitude to $V_{S D}$ in this scenario. For the increasing- $V_{G}$ sweeps (dashed curves), we observe conductivity minima at $V_{G} \approx 4 \mathrm{~V}$ and $V_{G}>4 \mathrm{~V}$ for the lower- and higher- $V_{S D}$ scenarios, respectively, where the end of the range is set to mitigate leakage current through the oxide. Accounting for the gate-potential-dependent photodoping, we conclude that fixed gate potentials of $V_{G}=4 \mathrm{~V}$ and $V_{G}=-16 \mathrm{~V}$ give $n$ - and $p$-type doping of the graphene ribbon, respectively, which should be maintained under subsequent laser pulsing (Appendix C), and hence proceed to acquire current-density maps under each of these conditions.

A PL image of the mapped device shows the $50-\mu \mathrm{m}$ wide graphene ribbon with a number of tears across the gated region, which arise during transfer and fabrication [Fig. 2(c)]. Throughout imaging we maintain a constant total injected current of $700 \mu \mathrm{A}$, giving $V_{S D}$ that varies around $15 \mathrm{~V}$. The reconstructed current-density maps of the GFET under $n$-type doping [Fig. 2(d), (i)] and $p$ type doping [Fig. 2(d), (ii)] show broadly similar features, where the current density is increased under the gated area as carriers are restricted to narrow passages due to the tears in the graphene. The difference in the current density magnitude at each pixel between the two doping conditions shows clear differences in the current path
[Fig. 2(e)]. Some of these variations are associated with degradation of the graphene channel throughout the measurement at $V_{G}=-16 \mathrm{~V}$; however, some are uncorrelated with changes to the graphene visible at our imaging resolution (Appendix D). Degradation of the devices throughout measurement precludes further investigation into the origin of these differences; however, in principle, such effects could arise from inhomogeneous doping of the graphene channel, gate-controlled steering of carriers [15], gateinduced changes to the density of charged impurities, and defects that have an asymmetric scattering cross section for electrons and holes [32-36].

\section{PL-SWITCHING EFFECT}

While varying the gate potential in the previous measurement, we observed that the total PL of the N- $V$ ensemble varied significantly with the applied gate potential. To investigate this effect, a measurement is performed in which the PL is accumulated under cw-laser illumination as the gate potential is varied. A settling time is introduced between setting the gate potential and starting the accumulation of the PL, which itself is integrated over a number of camera cycles to average out fluctuations in the illuminating-laser intensity. A small source-drain potential, $V_{S D}=100 \mathrm{mV}$, is applied to track the device conductivity throughout the measurement.

This measurement is done on the same device in which current densities were mapped. The gate potential is swept from 4 to $-16 \mathrm{~V}$ and back, encompassing the neutrality point of the device, which is seen at approximately $-12 \mathrm{~V}$ [Fig. 3(a)]. The device resistance is left to equilibrate at $V_{G}=4 \mathrm{~V}$ under laser illumination before sweeping. Comparison of snapshots of the accumulated PL images at the extrema of the gate-potential sweep [Fig. 3(b)] demonstrates a clear quenching of the PL near the graphene ribbon under the gate at the lower gate potential. Looking at area-normalized PL curves around the device, we see that the PL is constant above $V_{G}=-8 \mathrm{~V}$, then declines by up to $25 \%$ in regions close to, but not directly beneath, the graphene [Fig. 3(c)]. To map the extent of this effect, we take a normalized difference in PL averaged across a $2-\mathrm{V}$ window at either end of the sweep, $L_{-}$and $L_{+}$ in Fig. 3(c), and plot this difference across the full field of view, $\Delta L$ [Fig. 3(d)]. The normalized-PL-difference images show the PL-switching effect occurs only under the top gate, in regions not screened by the graphene. Surprisingly, this effect extends laterally from the graphene ribbon by up to $20 \mu \mathrm{m}$ [Fig. 3(e)]. The magnitude of this $\mathrm{PL}$ change increases throughout the lifetime of the device (Appendix C).

A likely explanation for this gate-potential-dependent PL is that varying the gate potential affects the charge distribution at the diamond-oxide interface, and hence the degree of band bending across the N- $V$ layer [37]. This 
(a)
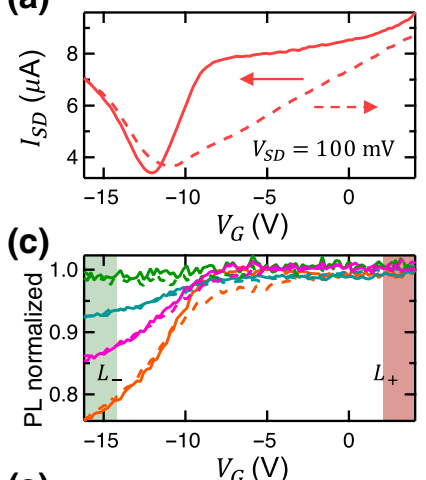

(e)

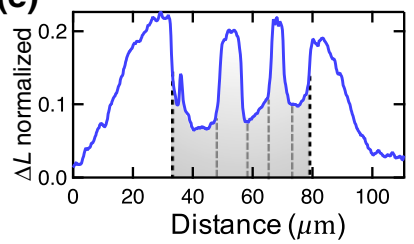

(b) $\quad \mathrm{PL}$ (arb. units)

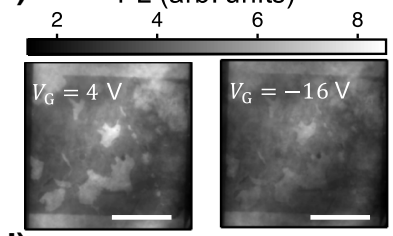

(d)

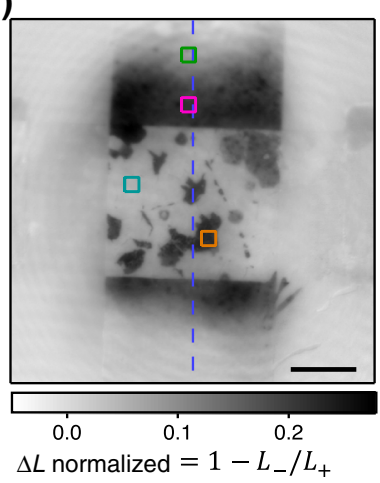

FIG. 3. Gate-potential-dependent PL. (a) $I_{S D}$ versus $V_{G}$ measured in conjunction with accompanying PL measurement. The sweep moves from high to low gate potential (solid line) and then reverses (dashed line). (b) PL images of the gated region of a GFET at $V_{G}=4 \mathrm{~V}$ and $V_{G}=-16 \mathrm{~V}$ showing quenched $\mathrm{PL}$ in the bare diamond proximal to the graphene at the lower gate potential. (c) Area-normalized PL curves from four regions surrounding the GFET, specifically, a tear in the gated graphene region (orange), pristine gated graphene (blue), and two regions under the gate at $5 \mu \mathrm{m}$ (pink) and $20 \mu \mathrm{m}$ (green) from the graphene-ribbon edge. Solid (dashed) lines shows PL measurements as the gate potential decreases (increases). Each sweep is normalized to the first data point at $V_{G}=4 \mathrm{~V}$. A settling time of $2 \mathrm{~s}$ with 50 camera cycles per data point is used for this sweep. (d) Normalized difference in PL across the GFET, where the plotted value is the difference between the integrated $\mathrm{PL}$ at high gate potential, $V_{G}=4$ to $2 \mathrm{~V}$ [band labeled " $L_{+}$" in (c)], and the PL at low gate potential, $V_{G}=-14$ to $-16 \mathrm{~V}$ [band labeled " $L_{-}$" in (c)], normalized by the value of $L_{+}$. The PL is averaged across both sweep directions. The colored squares indicate the areas for which the full PL curves are shown in (c). A settling time of 0.5 $\mathrm{S}$ with five camera cycles per data point is used for this sweep to minimize fringe artifacts from drifting optics. (e) Profile of the difference in normalized PL running across the graphene ribbon parallel to the gate [dashed blue line in (d)]. The edges of the graphene ribbon (tears) are indicated by the dashed black (gray) lines. All scale bars are $20 \mu \mathrm{m}$.

effect was investigated previously by variation of the surface chemistry of the diamond $[38,39]$ and by electrostatic gating of $\mathrm{N}-V$ centers $[19,40,41]$. Here we suggest that an increasing negative potential populates an acceptor layer at the diamond surface [42], given the low carrier density within the doped region, and hence increases the band bending across the $\mathrm{N}-V$ layer. As the band bending increases, the Fermi level falls below the $\mathrm{N}-V^{-}$charge state at greater depths within the diamond. Once this occurs around the mean depth of the $\mathrm{N}-V$ ensemble, the PL of the

layer is reduced significantly as $\mathrm{N}-V^{0}$ becomes the dominant charge state. The comparative abundance of charge carriers in the graphene is expected to screen this effect, and hence we observe only the PL switching close to the gated device, and not directly under the graphene ribbon. The charge-state stability and dynamics under illumination and in the dark may also be altered by the gate potential through the local charge environment within the diamond $[43,44]$.

\section{ELECTRIC FIELD MEASUREMENTS}

To test the hypothesis outlined, a direct measurement of the electric field is made. The electric field across the $\mathrm{N}-V$ layer can be measured in a direct and quantitative manner via the Stark effect on the N- $V$ spin states [1]. To do this, we perform an ODMR measurement, as for the previous Ørsted-field measurements, but with the biasing magnetic field oriented such that the magnetic field projection along a single $\mathrm{N}-V$ family is minimized, enhancing its electric field sensitivity $[37,45]$, but still allowing each spin-state transition of that family to be resolved [Fig. 4(a)]. The optical contrast of these transitions is selectively enhanced by rotation of the linear polarization of the excitation laser to further increase sensitivity [46]. The electric field is reconstructed under the assumption that only the surface normal (z) component contributes, as the lateral field components for a sheet of charge at or above the diamond average to zero in the $\mathrm{N}-V$ layer.

The electric field is mapped across a single GFET [Fig. 4(b)] for applied gate potentials $V_{G}=0 \mathrm{~V}$ and $V_{G}=$ $-12 \mathrm{~V}$ [Fig. 4(c)]. In both measurements, the electric field is approximately $40 \mathrm{kV} / \mathrm{cm}$ less in the $\mathrm{N}-V$ layer under the graphene ribbon than that directly under the oxide. This may be partially due to the graphene modifying the spacecharge distribution at the diamond surface, and hence decreasing the band bending; however, it is more likely to be fully explained by the FRET effect, with the graphene quenching PL from N- $V$ centers closer to the surface, biasing the PL collection to deeper $\mathrm{N}-V$ centers, which see a lower electric field. At $V_{G}=-12 \mathrm{~V}$, the electric field increases at the edges and within tears in the graphene ribbon under the top gate. These features are highlighted in a subtraction of the two maps [Fig. 4(d)], which shows an enhanced electric field up to $60 \mathrm{kV} / \mathrm{cm}$ in these regions, and essentially no change under the graphene ribbon.

Comparing the electric field map with the normalized difference in PL of the same device [Fig. 4(e)], we see a strong correlation in the location of features showing an enhanced electric field and reduced PL at low gate potential. The correspondence between the directly measured electric field and the gate-potential-dependent PL validates the claim that PL dependence on gate potential arises from electric-field-mediated band bending within the diamond [37]. The spatial distribution of this effect, 


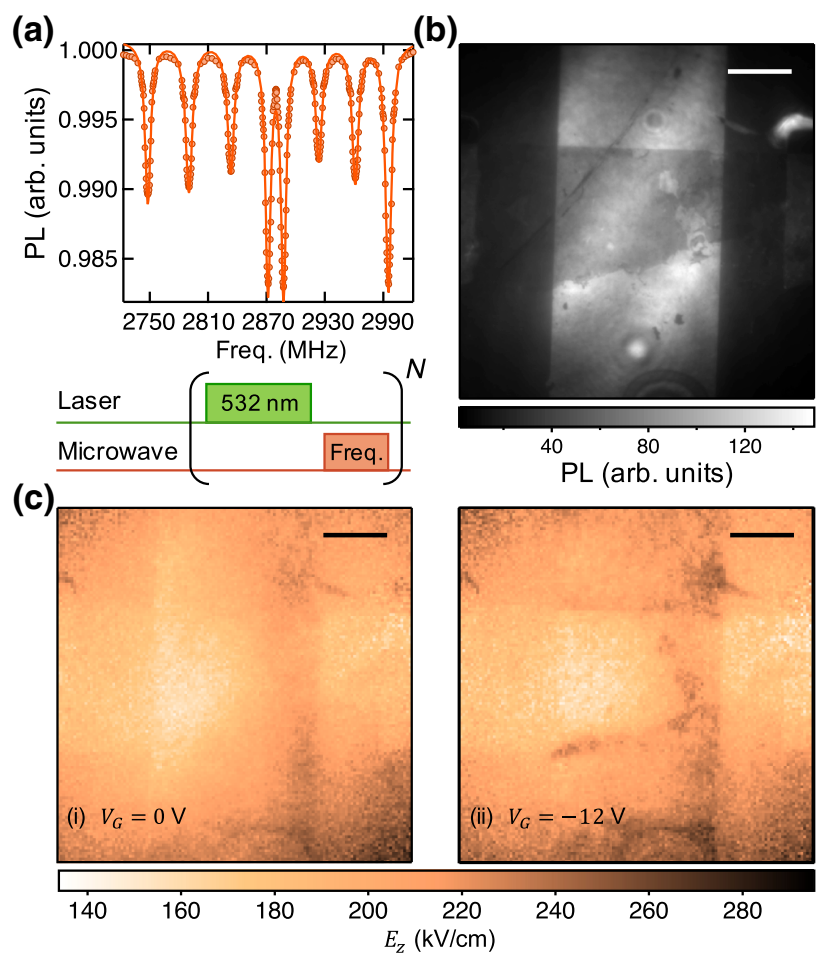

(d)

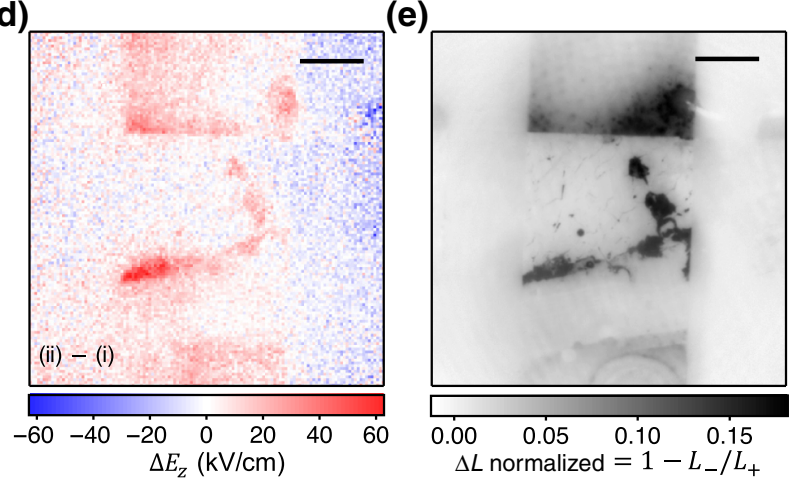

FIG. 4. Electric field measurements. (a) ODMR spectrum optimized for electric field sensing by minimization of the biasmagnetic-field projection along one $\mathrm{N}-V$ family and enhancement of the optical contrast (top). ODMR pulse sequence (bottom). (b) PL image of the electric-field-mapped device taken under $\mathrm{cw}$ illumination. (c) Maps of the electric field in the $z$ direction at two applied gate potentials, (i) $V_{G}=0 \mathrm{~V}$ and (ii) $V_{G}=-12 \mathrm{~V}$. The map is extracted from the ODMR data, presuming the field lies only in the $z$ direction for simplicity. A small source-drain potential is applied for each measurement $V_{S D}=100 \mathrm{mV}$ to track the conductivity of the device. (d) Map of the difference in electric fields measured at $V_{G}=-12 \mathrm{~V}$ and $V_{G}=0 \mathrm{~V}$. (e) Normalized difference in PL $(\Delta L)$ between the PL at high gate potential, $V_{G}=-1$ to $1 \mathrm{~V}\left(L_{+}\right)$and the PL at low gate potential, $V_{G}=-11$ to $-13 \mathrm{~V}\left(L_{-}\right)$normalized by $L_{+}$as in Fig. 3. The data are taken with a 0.5 -s settling time and five camera accumulations per gate-potential value. All scale bars are $20 \mu \mathrm{m}$.

namely, that it extends up to $20 \mu \mathrm{m}$ from the gated device, is less trivial. A finite-element-method simulation of the purely dielectric response of the system indicates that the electric field from the gate should be less than $10 \mathrm{kV} / \mathrm{cm}$ within the N- $V$ layer for distances greater than $5 \mu \mathrm{m}$ from the graphene edge, and thus cannot account for the observations (Appendix E). For this reason, we suggest that the measured electric field originates from trapped charge at the diamond-oxide interface that accumulates above a threshold gate potential. Although the exact mechanism is unclear, this accumulation may result from charge diffusion (Appendix C) either through the oxide or through the diamond itself, mediated by photoexcitation caused by the laser [47].

\section{OUTLOOK}

This work highlights the invasiveness of $\mathrm{N}-V$ microscopy in the case of GFETs fabricated directly on a $\mathrm{N}$ - $V$-diamond substrate. Namely, we find that control over the average doping in the graphene layer is strongly affected by a gatepotential-dependent photodoping effect, while degradation in the device from measurement at large gate potentials over timescales required for $\mathrm{N}-V$ imaging limits the ability to probe a single device in numerous scenarios. Additionally, we see evidence of a complex electrostatic response at the oxide-graphene and diamond-oxide interfaces that is not limited to atomic-layer-deposition $\mathrm{Al}_{2} \mathrm{O}_{3}$ dielectric [25-27], which raises the possibility of uncontrolled spatially dependent doping variations. For future applications of $\mathrm{N}-V$ microscopy where precise, reliable control over the doping is required, these effects must be mitigated. One possible solution is to decouple the GFET from the diamond by capping the diamond with a metal-oxide bilayer before fabricating the GFET. The extra metallic layer would prevent laser radiation from reaching the GFET [48], drastically reduce any charge-transfer effect at the diamond surface by providing an electron reservoir, and could even serve as a bottom gate for the GFET. A downside of this solution is that the graphene layer can no longer be visualized optically through the FRET effect. Further enhancements can made by encapsulating the graphene ribbon in hexagonal boron nitride, which allows the hexagonal boron nitride to be used as a gate dielectric, and is known to improve graphene quality $[13,17]$.

A remaining issue arises from the large source-drain currents typically required for $\mathrm{N}-V$ magnetometry. This requirement could be relaxed by increasing the sensitivity of the $\mathrm{N}-V$ sensing layer. In the present work, we aim for a mean graphene- $\mathrm{N}-V$ distance of only $10-20 \mathrm{~nm}$ to preserve a sizable FRET effect, facilitating imaging, but this requirement has a direct impact on sensitivity by limiting the maximum number of $\mathrm{N}-V$ centers without compromising the $\mathrm{N}-V$ spin coherence [49]. However, in principle, thicker $\mathrm{N}-V$ layers (e.g., $200 \mathrm{~nm}$ ) can be used without deteriorating the spatial resolution, which would remain limited by diffraction (approximately $300 \mathrm{~nm}$ ) [50]. For instance, the optimized $\mathrm{N}-V$ layer reported in Ref. [51] 
would provide a tenfold increase in magnetic sensitivity. With a further increase in collected PL signal due to the extra metallic layer, $\mathrm{N}-V$ measurements with sourcedrain currents in the microampere range can be envisaged. Additionally, the use of pulsed current, timed to align precisely with magnetic field measurement [24], would minimize current-induced degradation. Probing similar devices in this dynamic state, however, may further complicate interpretation of the measurements. Implementing these solutions may allow minimally invasive wide-field $\mathrm{N}-V$ microscopy of GFETs and other electrical devices based on two-dimensional materials.

\section{ACKNOWLEDGMENTS}

We thank Daniel J. McCloskey and Alastair Stacey for useful discussions. This work was supported by the Australian Research Council through Grants No. DE170100129, No. CE170100012, and No. FL130100119. This work was performed in part at the Melbourne Centre for Nanofabrication in the Victorian Node of the Australian National Fabrication Facility. D.A.B. and S.E.L. are supported by an Australian Government Research Training Program Scholarship.

\section{APPENDIX A: DIAMOND SAMPLES}

The $\mathrm{N}-V$-diamond samples used in these experiments are made from $4 \mathrm{~mm} \times 4 \mathrm{~mm} \times 50 \mu \mathrm{m}$ electronic grade ( $\mathrm{N}$ concentration less than $1 \mathrm{ppb}$ ) single-crystal diamond plates with $\{110\}$ edges and a (100) top facet purchased from Delaware Diamond Knives. The diamond surfaces are polished to a roughness of less than $2 \mathrm{~nm}$ [6]. The plates are laser-cut into smaller $2 \mathrm{~mm} \times 2 \mathrm{~mm} \times 50 \mu \mathrm{m}$ plates, acid-cleaned (15 min in a boiling mixture of sulfuric acid and sodium nitrate), and implanted with ${ }^{15} \mathrm{~N}^{+}$ ions (InnovIon) at an energy of $6 \mathrm{keV}$ and a fluence of $10^{13}$ ions $/ \mathrm{cm}^{2}$ with a tilt angle of $7^{\circ}$. This energy gives a mean implantation depth within the range of $10-$ $20 \mathrm{~nm}$ [49]. Following implantation, the diamonds are annealed in a vacuum of approximately $10^{-5}$ Torr to form the $\mathrm{N}-V$ centers with use of the following sequence: $6 \mathrm{~h}$ at $400{ }^{\circ} \mathrm{C}, 2 \mathrm{~h}$ ramp to $800^{\circ} \mathrm{C}, 6 \mathrm{~h}$ at $800^{\circ} \mathrm{C}, 2 \mathrm{~h}$ ramp to $1100^{\circ} \mathrm{C}, 2 \mathrm{~h}$ at $1100^{\circ} \mathrm{C}$, and $2 \mathrm{~h} \mathrm{ramp}$ to room temperature. To remove the graphitic layer formed during the annealing at the elevated temperatures, the samples are acid-cleaned (as before).

\section{APPENDIX B: FABRICATION}

The GFETs in this work all consist of monolayer polycrystalline graphene ribbons, $\mathrm{Cr} / \mathrm{Au}$ source-drain contacts, and an 80-nm $\mathrm{Al}_{2} \mathrm{O}_{3}$ gate oxide with a $\mathrm{Cr} / \mathrm{Au}$ top-gate contact. Two sets of ten GFETs are fabricated on two different $\mathrm{N}-V$-diamond substrates, labeled as "diamond no. 200" and "diamond no. 230." On diamond no. 200 the graphene ribbons are $50 \times 500 \mu \mathrm{m}^{2}$, with the source contacts evaporated on top of the graphene. On diamond no. 230 the ribbons are $20 \times 500 \mu \mathrm{m}^{2}$, and the graphene is transferred onto the top of already-existing contacts.

Graphene is transferred from commercially available (Graphenea) monolayer graphene grown by chemical vapor deposition on a copper foil by a standard wet chemical technique [21]. Before transfer, the graphene is spin-coated with a PMMA A4 (950) protective layer. The copper foil is etched in a $0.5 \mathrm{wt} \% \mathrm{Fe}\left(\mathrm{NO}_{3}\right)_{3}$ solution for $24 \mathrm{~h}$. The sample is transferred (with a clean Si wafer) through multiple deionized water rinses, a dilute RCA2 (1:1:10 34\% $\mathrm{HCl}-\mathrm{H}_{2} \mathrm{O}_{2}$-deionized $\mathrm{H}_{2} \mathrm{O}$ ) cleaning step, and further rinsing before being transferred to a $2 \times 2 \mathrm{~mm}^{2}$ $\mathrm{N}-V$-diamond substrate, mounted on a $300-\mathrm{nm} \mathrm{SiO}_{2} / \mathrm{Si}$ wafer. The sample is left to dry for $48 \mathrm{~h}$.

The $\mathrm{Al}_{2} \mathrm{O}_{3}$ top-gate oxide is grown by atomic layer deposition, with trimethylaluminum and water precursors at $200{ }^{\circ} \mathrm{C}$. Nucleation issues on the graphene are mitigated by increasing the water residence time with a double pulse within the first 50 cycles [22]. The bonding pads are exposed by etching of the oxide in an $8 \% \mathrm{NH}_{4} \mathrm{OH}$ solution at $50{ }^{\circ} \mathrm{C}$ for $25 \mathrm{~min}$.

Contacts, bonding pads, and the top gate are fabricated with photolithography using TI35E photoresist, followed by thermal electron-beam evaporation and lift-off of $\mathrm{Cr}(10$ $\mathrm{nm}) / \mathrm{Au}(70 \mathrm{~nm})$. The graphene is also patterned by photolithography, with use of SU8 (negative tone photoresist) on a protective PMMA layer to create a removable hard mask for etching in an oxygen plasma asher $(750 \mathrm{~W}$, $10-\mathrm{sccm} \mathrm{O}_{2}$ in Ar).

\section{APPENDIX C: ELECTRICAL CHARACTERIZATION AND PHOTODOPING}

The GFETs are characterized electrically throughout their lifetime to track their resistance and doping. $I_{S D^{-}}$ versus- $V_{S D}$ curves are recorded for all devices before imaging, showing reasonable Ohmic behavior of the graphene ribbons [Fig. 5(a)]. Linear fits of the $I_{S D}$-versus- $V_{S D}$ curves give resistances of $6.38,13.21$, and $6.49 \mathrm{k} \Omega$ for devices 1,2 , and 3 , respectively, on diamond no. 200. The device resistances typically increase throughout measurement, particularly after measurement at high source-drain currents (greater than $500 \mu \mathrm{A}$ ) and large gate potentials $\left(\left|V_{G}\right|>8 \mathrm{~V}\right)$, which are observed to cause tearing in the ribbon (Appendix D). Conductivity minima are also found for these devices before imaging, by measurement of $I_{S D}$ as a function of $V_{G}$ for a small source-drain potential, $V_{S D}=100 \mathrm{mV}$, under cw-laser illumination [Fig. 5(b)]. Devices 1, 2, and 3 on diamond no. 200 show conductivity minima at $V_{G} \approx-11 \mathrm{~V}$.

Throughout the course of imaging a device, $I_{S D^{-}}$ versus- $V_{G}$ curves are measured regularly under cw-laser 

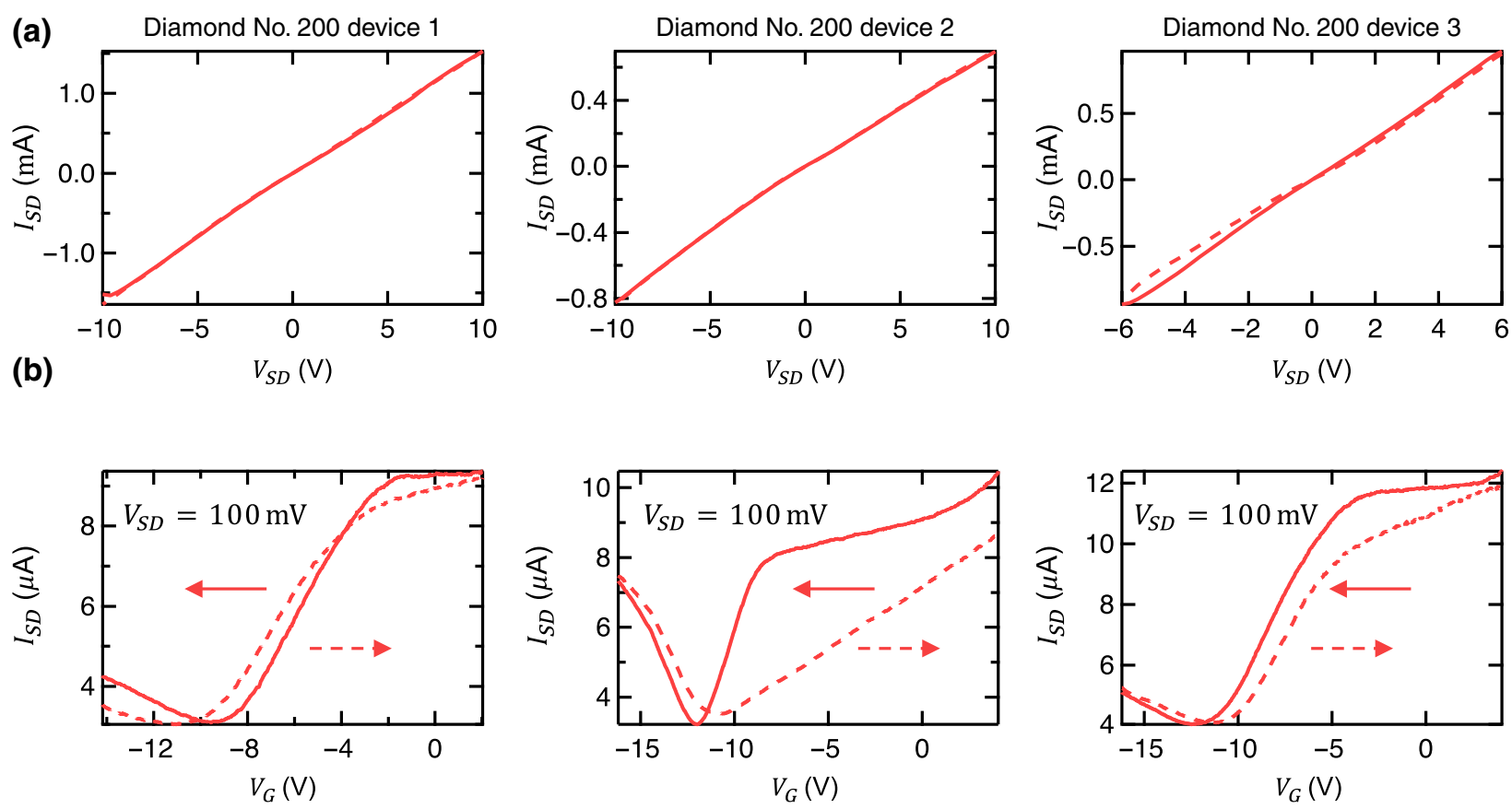

FIG. 5. Electrical characterization of GFETs. (a) $I_{S D}$ versus $V_{S D}$ for three devices on diamond no. 200. Averaging linear fits of the increasing and decreasing sweeps gives resistances of 6.38, 13.21, and $6.49 \mathrm{k} \Omega$ for devices 1,2 , and 3, respectively. Solid (dashed) lines indicate increasing (decreasing) $V_{S D}$ sweeps. (b) $I_{S D}$ versus $V_{G}$ showing neutrality points at $V_{G} \approx-11 \mathrm{~V}$ for the same three devices, measured with a small source-drain current, $I_{S D}=100 \mathrm{mV}$ under cw-laser illumination. Solid (dashed) lines indicate decreasing (increasing) $V_{G}$ sweeps. The device current is left to equilibrate at the initial point of each sweep before the gate potential is swept.

illumination to track changes in the effective doping of the device under the relevant imaging conditions. We observe that sustained imaging of the devices, which requires a prolonged exposure to some combination of laser, gate potential, and source-drain current, results in shifts of the conductivity minima, in addition to an enhanced hysteresis in the $I_{S D}$-versus- $V_{G}$ curves measured under laser illumination [Fig. 6(a)]. In conjunction with this effect, we observe enhanced quenching of the N- $V$ PL at low gate potentials over the same time frame [Fig. 6(b)]. The normalizeddifference-in-PL maps shown are produced in the same fashion as those presented in Fig. 3, and demonstrate an increase in the magnitude of the PL change, and its lateral extent, after prolonged imaging. Importantly, the onset of this effect occurs consistently at a threshold gate potential of $-8 \mathrm{~V}$.

An explanation for these changes to the effective device doping and enhanced hysteresis is that there is a photonassisted charge transfer between the graphene and the oxide, similar to the optical doping seen with other gate dielectrics and substrates [25-27], which has some dependence on the applied gate potential at the time of illumination. To test this, a single device is exposed to $\mathrm{cw}$-laser illumination under a fixed gate potential $\left(V_{G}^{\mathrm{PD}}\right)$ and left to equilibrate for $6 \mathrm{~min}$, while a small source-drain potential, $V_{S D}=100 \mathrm{mV}$, is applied to measure the current through the device. The laser is then turned off, and then the gate potential is swept between -10 and $10 \mathrm{~V}$ while the source-drain current is measured to identify the conductivity minimum $\left(V_{G}^{\mathrm{min}}\right)$. The measurement is repeated for each photodoping gate potential to measure the transport curve by sweeping the gate potential in the opposite direction, which is seen to systematically shift the location of the minima. We attribute this to the presence of stray light incident on the sample unpinning the photodoping during the sweep, and additional trapped charge at the interfaces. The sample is photodoped at $V_{G}^{\mathrm{PD}}=0 \mathrm{~V}$ between each measurement, such that subsequent photodoping proceeds from similar initial conditions.

The conductivity minima are extracted from transport measurements for $V_{G}^{\mathrm{PD}}$ between -10 and $10 \mathrm{~V}$ [Fig. 7(a)]. Photodoping at $V_{G}^{\mathrm{PD}}=0 \mathrm{~V}$ gives an $n$-typedoped graphene channel with conductivity minimum at $V_{G}^{\min } \approx-5 \mathrm{~V}$. The minimum shifts to higher gate potentials as $V_{G}^{\mathrm{PD}}$ is increased, giving a $p$-type device for $V_{G}^{\mathrm{PD}} \geq 8$. At negative $V_{G}^{\mathrm{PD}}$, we identify a strong threshold at $V_{G}^{\mathrm{PD}}=-6 \mathrm{~V}$, where the conductivity minimum shifts to positive gate potentials, $V_{G}^{\min }>10 \mathrm{~V}$, a regime we are unable to measure due to large leakage currents through the gate oxide. This threshold closely corresponds to the onset of the PL-switching effect, and hence suggests that both phenomena may be due an accumulation of holes within the oxide and at the diamond-oxide and graphene-oxide interfaces. The full transport curves 

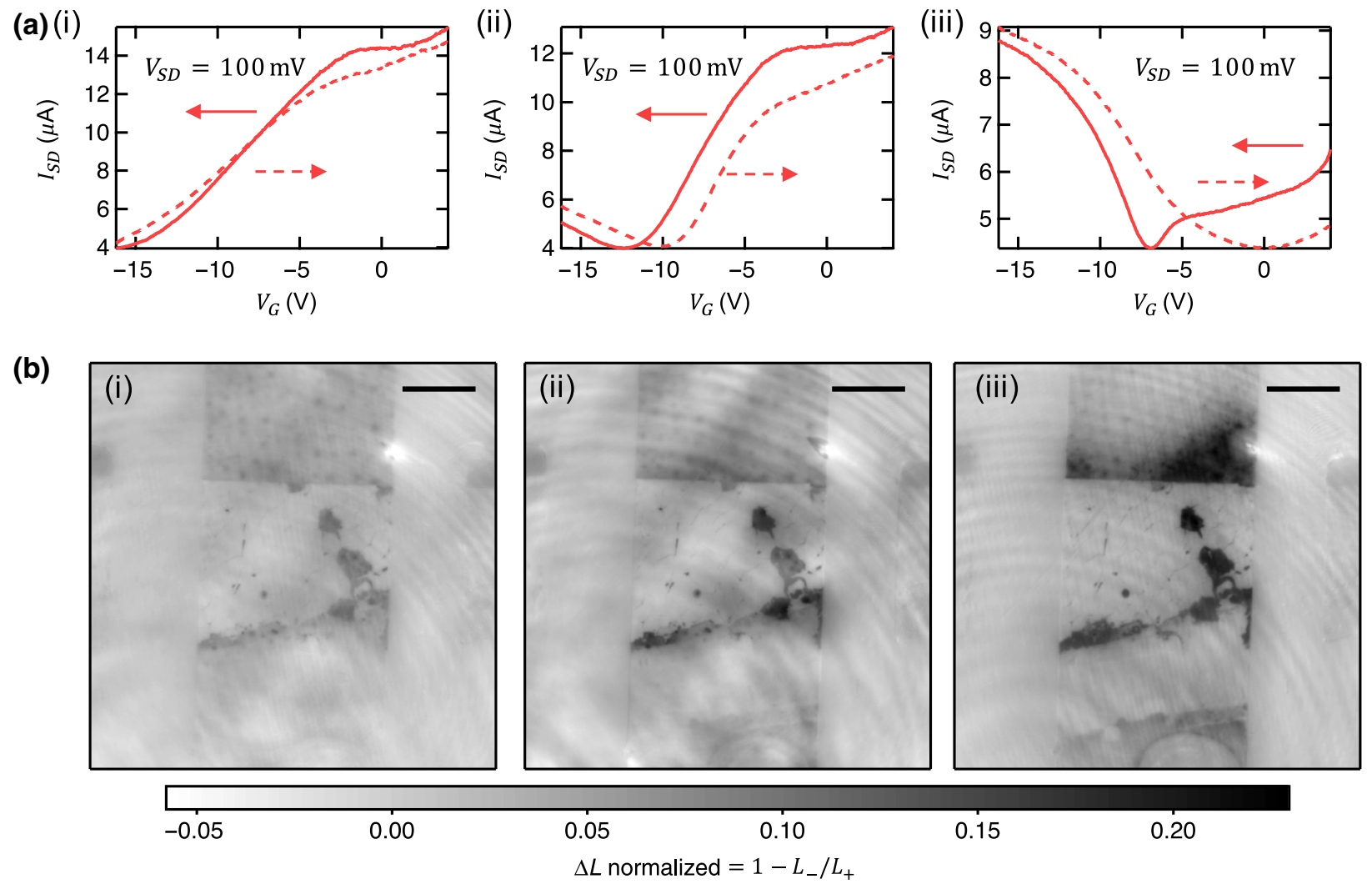

FIG. 6. Evolution of neutrality-point- and gate-potential-dependent PL. (a) $I_{S D}$-versus- $V_{G}$ curves for device 3 on diamond no. 200 : (i) before any sustained measurements, (ii) after electric field mapping at $V_{G}=0 \mathrm{~V}$ and $V_{G}=-8 \mathrm{~V}$, and (iii) after electric field mapping at $V_{G}=-12 \mathrm{~V}$. All measurements are taken under cw-laser illumination with $V_{S D}=100 \mathrm{mV}$. (b) (i)-(iii) Normalized-difference-inPL maps of device 3 on diamond no. 200 taken immediately after the measurements in (a). The plotted value is the difference between the integrated PL at high gate potential, $V_{G}=2$ to $4 \mathrm{~V}\left(L_{+}\right)$, and the PL at low gate potential, $V_{G}=-14$ to $-16 \mathrm{~V}\left(L_{-}\right)$, normalized by $L_{+}$. The PL is averaged across both sweep directions. A settling time of $2.0 \mathrm{~s}$ with 50 camera cycles per data point is used for this sweep. All scale bars are $20 \mu \mathrm{m}$.

measured with increasing-gate-potential sweeps following different photodoping gate potentials highlight this effect [Fig. 7(b)], where the conductivity minima are beyond the range of the sweep for photodoping $V_{G}^{\mathrm{PD}} \leq-8 \mathrm{~V}$.

The timescales over which the photodoping of the graphene channel occurred are measured by our tracking the source-drain current within the 6-min window during which the cw-laser is switched on or off and the gate potential is set [Fig. 7(c)]. The time evolution of $I_{S D}$ at $V_{G}=0 \mathrm{~V}$ (green curve) demonstrates the necessity of the laser for the photodoping effect, which initiates a decrease in conductivity of the device over minute-long timescales from some initially pinned value. The gate-potential dependence is evident in the time trace when the gate potential $V_{G}$ is changed from 0 to $10 \mathrm{~V}$ (purple curve) and from 0 to $-10 \mathrm{~V}$ (orange curve) under cw-laser illumination, after having equilibrated under laser illumination at $V_{G}^{\mathrm{PD}}=0 \mathrm{~V}$. As the gate potential is changed, there is a large change in conductivity, as the system jumps to a doping level dictated by the $I_{S D}$-versus- $V_{G}$ curve for photodoping at $V_{G}^{\mathrm{PD}}=0 \mathrm{~V}$, and then a slower evolution of the conductivity, which in each case corresponds to a shifting of the conductivity minimum to higher gate potentials and hence reduction (increase) of $I_{S D}$ in the $V_{G}=10 \mathrm{~V}(-10 \mathrm{~V})$ case. The time evolution of $I_{S D}$ is best fit by a biexponential with a fast and slow component acting on timescales of 10 and 100 $\mathrm{s}$, respectively, the exact values of which are sensitive to the initial conditions of the photodoping. We also observe a jump in the conductivity of the device when the laser is turned off after the device has equilibrated under $\mathrm{cw}$ illumination (seen at $280 \mathrm{~s}$, orange curve), suggesting a gate dielectric dynamic faster than our time resolution $(0.08 \mathrm{~s})$; however, the system equilibrates to a similar doping level as reached under illumination. For this reason, we conclude that the doping achieved by a set gate potential under $\mathrm{cw}$-laser illumination is maintained when the illumination is then pulsed as required for $\mathrm{N}-V$ measurements such as ODMR measurements. Time traces of the device sourcedrain current throughout ODMR measurements endorse this. 

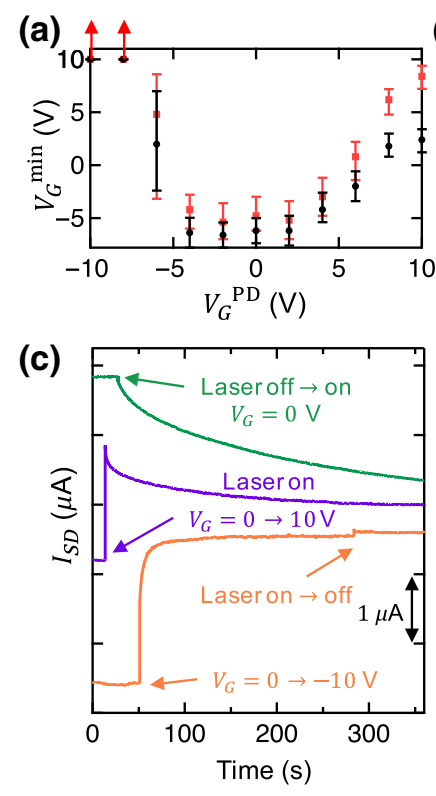

(b)

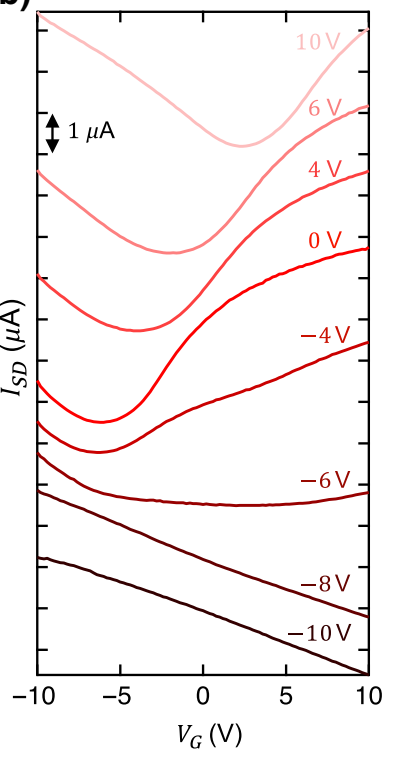

FIG. 7. Photodoping of a single GFET. (a) Measured gate potential of the conductivity minimum, $V_{G}^{\min }$, as a function of the gate potential applied during photodoping, $V_{G}^{\mathrm{PD}} . V_{G}^{\mathrm{min}}$ is given as measured from increasing- $V_{G}$ (black) and decreasing- $V_{G}$ (red) sweeps. The error bars show the width of the transport curve at $0.1 \mu \mathrm{A}$ from the conductivity minimum. (b). Individual $I_{S D^{-}}$ versus- $V_{G}$ curves for a single GFET as measured in the dark following photodoping at the labeled gate potential, $V_{G}^{\mathrm{PD}}$. The curves are all measured with increasing-gate-potential sweeps, and correspond to the red data set shown in (a). The curves are offset for clarity, and the minimum $I_{S D}$ values for each measurement are $2.69,3.10,3.22,3.01,3.78,4.49,3.79$, and $4.39 \mu \mathrm{A}$ in descending order of the photodoping gate potentials. (c) Time traces of $I_{S D}$ show the effect of photodoping parameters. $V_{G}=$ $0 \mathrm{~V}$ for the entirety of the green curve, and the laser is turned on at the indicated time point, leading to a biexponential decay in the device current. The $\mathrm{cw}$ laser is on initially for the purple (orange) curves, and the gate potential $V_{G}$ is switched from 0 to $10 \mathrm{~V}(-10 \mathrm{~V})$. The laser is turned off in the orange time trace at approximately $280 \mathrm{~s}$. The minimum current in each time trace is $3.20,3.39$, and $2.81 \mu \mathrm{A}$ for the green, purple, and orange curves respectively.

\section{APPENDIX D: CURRENT-DENSITY RECONSTRUCTION}

The current-density maps presented in the main text are produced by a method established in previous work [10, 24], which proceeds in the following manner. The ODMR spectrum at each pixel is fit with an eight-Lorentzian sum, and the frequencies are extracted. The magnetic field projection along the four $\mathrm{N}-V$-family orientations is calculated from the Zeeman splitting of each frequency pair from the zero-field resonance at $2870 \mathrm{MHz}$. The field is then converted to Cartesian coordinates with use of the three most-split $\mathrm{N}-V$ families, their orientation relative to the diamond surface having previously been determined by measurement of a field of known orientation $[52,53]$.
To reconstruct the current density from the measured magnetic field, we invert the Biot-Savart law in Fourier space [54,55]. Here we take only the $B_{z}$ component of the measured magnetic field and linearly extrapolate the remnant field in the $y$ direction to minimize truncation artifacts in the Fourier transform [24]. The Fourier-space current densities in the $x$ and $y$ directions are calculated trivially from the transformed $B_{z}$, and their inverse Fourier transform gives us the real-space densities [24].

Previous work has highlighted an apparent delocalization of current from metallic systems on the diamond surface to the diamond itself, as measured by this same technique [24]. All current densities plotted in this work represent the total reconstructed current density (above and below the N- $V$ layer), but most of the current is found to lie above the measuring N- $V$ layer. This scenario is consistent as the gate potential is varied.

Each current-density map produced arises from two separate ODMR measurements of the device at the given gate potential: one with and the other without the injected source-drain current. This is done to account for any magnetic or electric field features not associated with the carrier transport in the GFET. The subtraction of the background measurement from the signal is performed before conversion of the $\mathrm{N}-V$-family field projections to Cartesian coordinates.

The current-density maps produced by this method show differing density distributions for each doping condition (Fig. 2). A simple explanation for these differences is that they are due to degradation of the graphene throughout the measurement, which is often observed following sustained high source-drain currents (more than $500 \mu \mathrm{A}$ ) and at large gate potentials $\left(\left|V_{G}\right|>8 \mathrm{~V}\right)$. To determine whether the observed differences arise from significant tearing of the graphene, we compare PL images of the device taken under the same conditions before mapping at $V_{G}=4 \mathrm{~V}$ and after mapping at $V_{G}=-16 \mathrm{~V}$ [Fig. 8(a)]. A subtraction of these two PL images highlights two regions (marked "1" and " 2 ") in the gated section of the graphene ribbon where the graphene is no longer visible via FRET interaction with the $\mathrm{N}-V$ layer in the later measurement [Fig. 8(b)]. PL imaging between the two current measurements indicates that these changes occurred during the $V_{G}=-16 \mathrm{~V}$ measurement. The fringes visible across the device in the subtraction are due to a slight shift in the optics between measurements.

Comparison of the PL subtraction with the currentdensity subtraction [Fig. 8(c)] shows that there is a deviation in the current path close to one of the tears (marked "1"). The region showing the most-distinct change in the current path (marked " 3 ") shows very little PL change, indicating that it is not associated with a degradation of the graphene visible at our imaging resolution. Further investigation into the cause of this current 

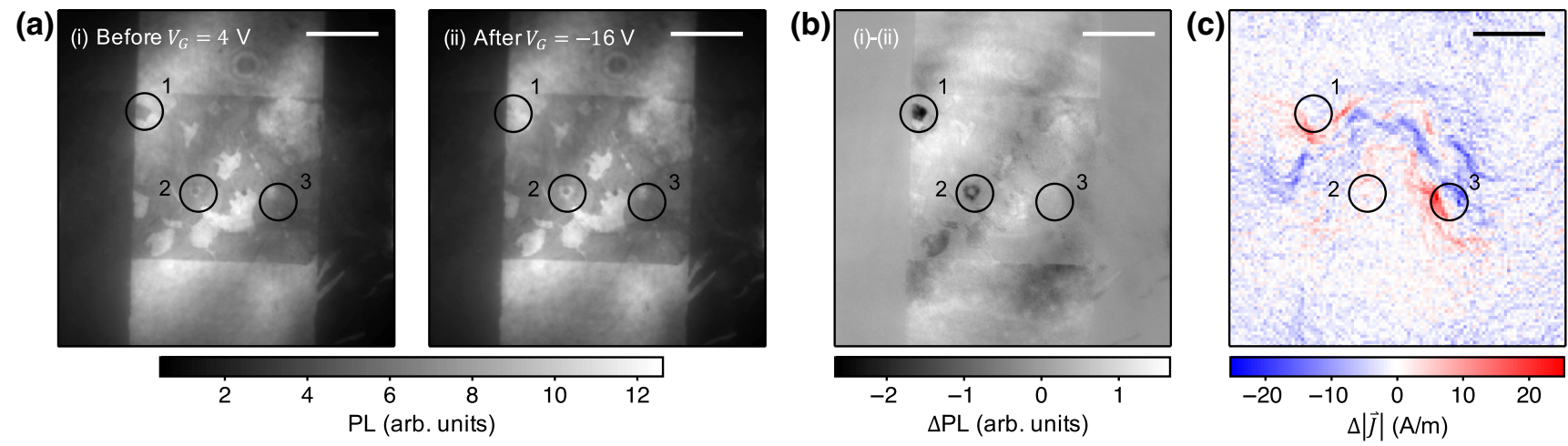

FIG. 8. PL imaging throughout current-density mapping. (a) PL images of the current-density-mapped GFET at $V_{G}=0 \mathrm{~V}$ taken (i) before mapping at $I_{S D}=700 \mu \mathrm{A}$ and $V_{G}=4 \mathrm{~V}$ and (ii) after mapping at $I_{S D}=700 \mu \mathrm{A}$ and $V_{G}=-16 \mathrm{~V}$. (b) Subtraction of (i) and (ii). (c) Difference in current density magnitude under doping conditions $V_{G}=4 \mathrm{~V}$ and $V_{G}=-16 \mathrm{~V}$ for comparison with the subtracted-PL map. All scale bars are $20 \mu \mathrm{m}$.

deviation is made difficult by the gradual deterioration of the device throughout measurement, which precludes repeated measurements, and provides motivation for a new generation of devices that better isolate the graphene from the diamond substrate and oxide-interface.

\section{APPENDIX E: ELECTRIC FIELD SIMULATIONS}

To determine the spatial distribution of the electric field from the top gate, such that it can be compared with that measured by the N- $V$ layer, a finite-element-method simulation is performed with COMSOL MULTIPHYSICS. The device geometry is replicated, where a $50-\mu \mathrm{m}$-wide metallic top gate is separated from a graphene ribbon by $80 \mathrm{~nm}$ of $\mathrm{Al}_{2} \mathrm{O}_{3}$, all hosted on top of a 50- $\mu$ m-thick diamond substrate. The electric field distribution is calculated in a shell surrounding the device, with use of a high-density mesh in the region of interest between the metallic planes and beneath the graphene plane, across the region containing the $\mathrm{N}-V$ layer.

The simulated electric field shows a high field strength between the parallel-plate capacitor $(-2 \mathrm{MV} / \mathrm{cm}$ in the center of oxide), which is screened from the diamond by the graphene plate [Fig. 9(a)]. Appreciable field strengths exist in the diamond only at the edge of the graphene ribbon under the top gate, where the magnitude reaches $-0.9 \mathrm{MV} / \mathrm{cm}$ in the $z$ direction, but reverses sign within a 300-nm length scale across the graphene-ribbon edge [Fig. 9(b)]. The component perpendicular to the graphene ribbon reaches $-1.2 \mathrm{MV} / \mathrm{cm}$ at the edge, but is laterally confined to $200 \mathrm{~nm}$. Given the optical-resolution-limited imaging via the $\mathrm{N}-V$-layer PL, we do not expect to be able to resolve these features, and hence conclude that the $\mathrm{N}-V$ layer measurements should not be sensitive to the electric field from the gate directly. Therefore, we propose that the enhanced electric field we measure, which correlates strongly with the gate-potential-dependent PL effect, must result from a change in the surface charge distribution at the diamond-oxide interface.

(a)
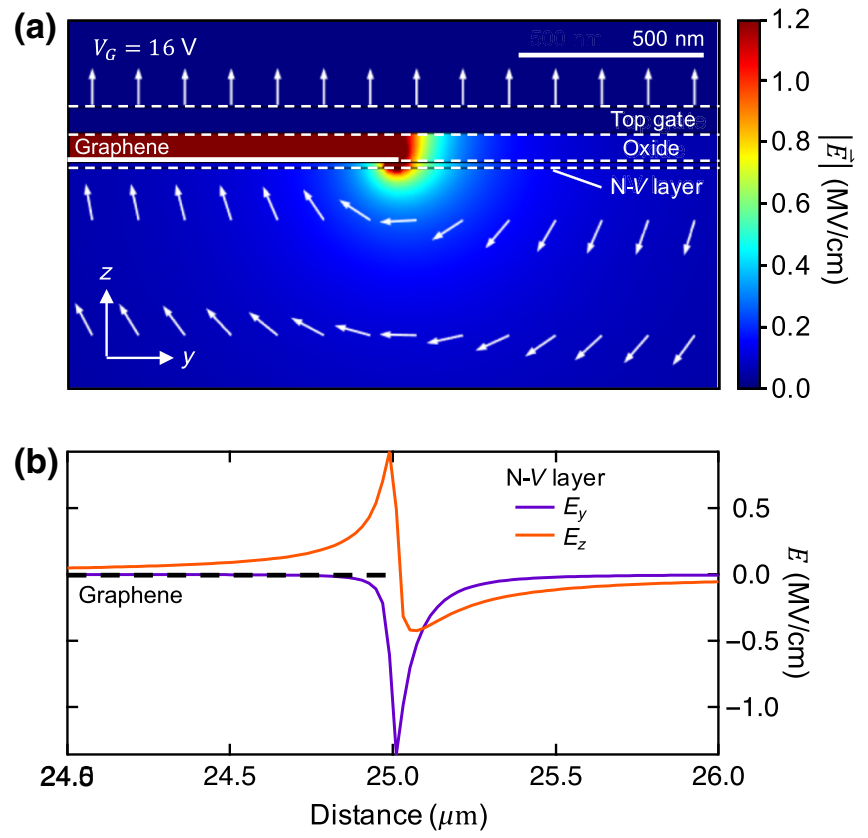

FIG. 9. COMSOL MULTIPHYSICS simulation of top-gate electric field. (a) Simulated-electric-field-magnitude plot for $V_{G}=16 \mathrm{~V}$ within a plane running perpendicular to the graphene-ribbon edge, in the middle of the top gate (i.e., corresponding to a vertical line cut of the images shown in the main text). The graphene extends from the left-hand side to the middle of the plot, whereas the top gate, oxide, and diamond cover the full region. The arrows show the projection of the electric field in the plane. The aspect ratio is $1: 1$, and the color scale is capped at $1.2 \mathrm{MV} / \mathrm{cm}$ to highlight features at lower field magnitudes. (b) Electric field components in the $z$ and $y$ directions in the $\mathrm{N}-V$ layer $(15 \mathrm{~nm}$ below the diamond surface) at $V_{G}=16 \mathrm{~V}$ across the width of the image shown in (a). The graphene-ribbon edge lies at $25 \mu \mathrm{m}$. 
[1] M. W. Doherty, N. B. Manson, P. Delaney, F. Jelezko, J. Wrachtrup, and L. C. L. Hollenberg, The nitrogen-vacancy colour centre in diamond, Phys. Rep. 528, 1 (2013).

[2] F. Casola, T. Van der Sar, and A. Yacoby, Probing condensed matter physics with magnetometry based on nitrogen-vacancy centres in diamond, Nat. Rev. Mater. 3, 17088 (2018).

[3] L. Rondin, J.-P. Tetienne, T. Hingant, J. F. Roch, P. Maletinsky, and V. Jacques, Magnetometry with nitrogenvacancy defects in diamond, Rep. Prog. Phys. 77, 056503 (2014).

[4] S. Kolkowitz, A. Safira, A. A. High, R. C. Devlin, S. Choi, Q. P. Unterreithmeier, D. Patterson, A. S. Zibrov, V. E. Manucharyan, H. Park, and M. D. Lukin, Probing Johnson noise and ballistic transport in normal metals with a single-spin qubit, Science 347, 1129 (2015).

[5] A. Ariyaratne, D. Bluvstein, B. A. Myers, and A. C. B. Jayich, Nanoscale electrical conductivity imaging using a nitrogen-vacancy center in diamond, Nat. Commun. 9, 2406 (2018).

[6] S. E. Lillie, D. A. Broadway, N. Dontschuk, A. Zavabeti, D. A. Simpson, T. Teraji, T. Daeneke, L. C. L. Hollenberg, and J.-P. Tetienne, Magnetic noise from ultra-thin abrasively deposited materials on diamond, Phys. Rev. Mater. 2, 116002 (2018).

[7] K. Agarwal, R. Schmidt, B. Halperin, V. Oganesyan, G. Zaránd, M. D. Lukin, and E. Demler, Magnetic noise spectroscopy as a probe of local electronic correlations in two-dimensional systems, Phys. Rev. B 95, 155107 (2017).

[8] J. F. Rodriguez-Nieva, K. Agarwal, T. Giamarchi, B. I. Halperin, M. D. Lukin, and E. Demler, Probing onedimensional systems via noise magnetometry with single spin qubits, Phys. Rev. B 98, 195433 (2018).

[9] K. Chang, A. Eichler, J. Rhensius, L. Lorenzelli, and C. L. Degen, Nanoscale imaging of current density with a singlespin magnetometer, Nano Lett. 17, 2367 (2017).

[10] J.-P. Tetienne, N. Dontschuk, D. A. Broadway, A. Stacey, D. A. Simpson, and L. C. L. Hollenberg, Quantum imaging of current flow in graphene, Sci. Adv. 3, e1602429 (2017).

[11] D. A. Bandurin, M. Ben Shalom, A. Principi, M. Polini, R. K. Kumar, I. V. Grigorieva, E. Khestanova, A. Tomadin, I. Torre, K. S. Novoselov, L. A. Ponomarenko, G. H. Auton, and A. K. Geim, Negative local resistance caused by viscous electron backflow in graphene, Science 351, 1055 (2016).

[12] K. A. Guerrero-Becerra, F. M. Pellegrino, and M. Polini, Magnetic hallmarks of viscous electron flow in graphene, Phys. Rev. B 99, 041407(R) (2019).

[13] M. J. H. Ku, T. X. Zhou, Q. Li, Y. J. Shin, J. K. Shi, C. Burch, H. Zhang, F. Casola, T. Taniguchi, K. Watanabe, P. Kim, A. Yacoby, and R. L. Walsworth, Imaging viscous flow of the Dirac fluid in graphene using a quantum spin magnetometer, arXiv:1905.10791 (2019).

[14] J. Martin, N. Akerman, G. Ulbricht, T. Lohmann, J. H. Smet, K. Von Klitzing, and A. Yacoby, Observation of electron-hole puddles in graphene using a scanning singleelectron transistor, Nat. Phys. 4, 144 (2008).
[15] J. R. Williams, T. Low, M. S. Lundstrom, and C. M. Marcus, Gate-controlled guiding of electrons in graphene, Nat. Nanotechnol. 6, 222 (2011).

[16] P. Kim, B. Ozyilmaz, M. Y. Han, A. F. Young, K. L. Shepard, and I. Meric, Current saturation in zero-bandgap, topgated graphene field-effect transistors, Nat. Nanotechnol. 3, 654 (2008).

[17] T. I. Andersen, B. L. Dwyer, J. D. Sanchez-Yamagishi, J. F. Rodriguez-Nieva, K. Agarwal, K. Watanabe, T. Taniguchi, E. A. Demler, P. Kim, H. Park, and M. D. Lukin, Electronphonon instability in graphene revealed by global and local noise probes, Science 364, 154 (2019).

[18] B. Hähnlein, B. Händel, J. Pezoldt, H. Töpfer, R. Granzner, and F. Schwierz, Side-gate graphene field-effect transistors with high transconductance, Appl. Phys. Lett. 101, 093504 (2012).

[19] M. V. Hauf, P. Simon, N. Aslam, M. Pfender, P. Neumann, S. Pezzagna, J. Meijer, J. Wrachtrup, M. Stutzmann, F. Reinhard, and J. A. Garrido, Addressing single nitrogenvacancy centers in diamond with transparent in-plane gate structures, Nano Lett. 14, 2359 (2014).

[20] Y. Ohno, K. Maehashi, Y. Yamashiro, and K. Matsumoto, Electrolyte-gated graphene FET for dectecting $\mathrm{pH}$ and protein adsorption, Nano Lett. 9, 3318 (2009).

[21] X. Liang, B. A. Sperling, I. Calizo, G. Cheng, C. Ann, Q. Zhang, Y. Obeng, K. Yan, H. Peng, Q. Li, X. Zhu, A. R. H. Walker, Z. Liu, L.-M. Peng, C. A. Richter, C. A. Hacker, H. Yuan, and A. R. Hight Walker, Towards clean and crackless transfer of graphene towards clean and crackless transfer of graphene, ACS Nano 5, 9144 (2011).

[22] A. I. Aria, K. Nakanishi, L. Xiao, P. Braeuninger-Weimer, A. A. Sagade, J. A. Alexander-Webber, and S. Hofmann, Parameter space of atomic layer deposition of ultrathin oxides on graphene, ACS Appl. Mater. Interfaces 8, 30564 (2016).

[23] J. Tisler, T. Oeckinghaus, R. J. Stöhr, R. Kolesov, R. Reuter, F. Reinhard, and J. Wrachtrup, Single defect center scanning near-field optical microscopy on graphene, Nano Lett. 13, 3152 (2013).

[24] J.-P. Tetienne, N. Dontschuk, D. A. Broadway, S. E. Lillie, T. Teraji, D. A. Simpson, A. Stacey, and L. C. L. Hollenberg, Apparent delocalization of the current density in metallic wires observed with diamond nitrogen-vacancy magnetometry, Phys. Rev. B 99, 014436 (2019).

[25] Y. D. Kim, M. H. Bae, J. T. Seo, Y. S. Kim, H. Kim, J. H. Lee, J. R. Ahn, S. W. Lee, S. H. Chun, and Y. D. Park, Focused-laser-enabled p-n junctions in graphene field-effect transistors, ACS Nano 7, 5850 (2013).

[26] P. Landois, M. Mikolasek, J. L. Sauvajol, M. Paillet, A. Tiberj, S. Contreras, J. R. Huntzinger, M. Rubio-Roy, E. Dujardin, and A. A. Zahab, Reversible optical doping of graphene, Sci. Rep. 3, 02355 (2013).

[27] L. Ju, J. Velasco, E. Huang, S. Kahn, C. Nosiglia, H. Z. Tsai, W. Yang, T. Taniguchi, K. Watanabe, Y. Zhang, G. Zhang, M. Crommie, A. Zettl, and F. Wang, Photoinduced doping in heterostructures of graphene and boron nitride, Nat. Nanotechnol. 9, 348 (2014).

[28] Y. G. Lee, C. G. Kang, U. J. Jung, J. J. Kim, H. J. Hwang, H. J. Chung, S. Seo, R. Choi, and B. H. Lee, Fast transient charging at the graphene $/ \mathrm{SiO}_{2}$ interface causing hysteretic device characteristics, Appl. Phys. Lett. 98, 183508 (2011). 
[29] D.-C. Mao, S.-Q. Wang, S.-A. Peng, D.-Y. Zhang, J.-Y. Shi, X.-N. Huang, M. Asif, and Z. Jin, The two timescales in the charge trapping mechanism for the hysteresis behavior in graphene field effect transistors, J. Mater. Sci.: Mater. Electron. 27, 9847 (2016).

[30] B. Krishna Bharadwaj, H. Chandrasekar, D. Nath, R. Pratap, and S. Raghavan, Intrinsic limits of channel transport hysteresis in graphene- $\mathrm{SiO}_{2}$ interface and its dependence on graphene defect density, J. Phys. D: Appl. Phys. 49, 265301 (2016).

[31] T. J. Ha, Visible-light-induced instability in amorphous metal-oxide based TFTs for transparent electronics, AIP Adv. 4, 107136 (2014).

[32] S. Adam, E. H. Hwang, V. Galitski, and S. D. Sarma, A selfconsistent theory for graphene transport, PNAS 104, 18392 (2007).

[33] E. H. Hwang, S. Adam, and S. Das Sarma, Carrier Transport in Two-Dimensional Graphene Layers, Phys. Rev. Lett. 98, 186806 (2007).

[34] T. O. Wehling, S. Yuan, A. I. Lichtenstein, A. K. Geim, and M. I. Katsnelson, Resonant Scattering by Realistic Impurities in Graphene, Phys. Rev. Lett. 105, 056802 (2010).

[35] L. C. Campos, I. Silvestre, A.-M. B. Goncalves, R. G. Lacerda, A. R. Cadore, M. S. C. Mazzoni, H. Chacham, A. S. Ferlauto, E. A. de Morais, and A. O. Melo, Asymmetric effect of oxygen adsorption on electron and hole mobilities in bilayer graphene: Long- and short-range scattering mechanisms, ACS Nano 7, 6597 (2013).

[36] K. K. Bai, Y. C. Wei, J. B. Qiao, S. Y. Li, L. J. Yin, W. Yan, J. C. Nie, and L. He, Detecting giant electron-hole asymmetry in a graphene monolayer generated by strain and charged-defect scattering via Landau level spectroscopy, Phys. Rev. B 92, 121405(R) (2015).

[37] D. A. Broadway, N. Dontschuk, A. Tsai, S. E. Lillie, C. T. Lew, J. C. Mccallum, B. C. Johnson, M. W. Doherty, A. Stacey, L. C. L. Hollenberg, and J.-P. Tetienne, Spatial mapping of band bending in a diamond device using in situ quantum sensors, Nat. Electron. 1, 502 (2018).

[38] M. V. Hauf, B. Grotz, B. Naydenov, M. Dankerl, S. Pezzagna, J. Meijer, F. Jelezko, J. Wrachtrup, M. Stutzmann, F. Reinhard, and J. A. Garrido, Chemical control of the charge state of nitrogen-vacancy centers in diamond, Phys. Rev. B 83, 081304(R) (2011).

[39] S. Cui and E. L. Hu, Increased negatively charged nitrogenvacancy centers in fluorinated diamond, Appl. Phys. Lett. 103, 051603 (2013).

[40] B. Grotz, M. V. Hauf, M. Dankerl, B. Naydenov, S. Pezzagna, J. Meijer, F. Jelezko, J. Wrachtrup, M. Stutzmann, F. Reinhard, and J. A. Garrido, Charge state manipulation of qubits in diamond, Nat. Commun. 3, 729 (2012).

[41] M. Pfender, N. Aslam, P. Simon, D. Antonov, G. Thiering, S. Burk, F. Fávaro De Oliveira, A. Denisenko, H. Fedder, J. Meijer, J. A. Garrido, A. Gali, T. Teraji, J. Isoya, M. W. Doherty, A. Alkauskas, A. Gallo, A. Grüneis, P. Neumann, and J. Wrachtrup, Protecting a diamond quantum memory by charge state control, Nano Lett. 17, 5931 (2017).

[42] A. Stacey, N. Dontschuk, J.-P. Chou, D. A. Broadway, A. Schenk, M. J. Sear, J.-P. Tetienne, A. Hoffman, S. Prawer, C. I. Pakes, A. Tadich, N. P. de Leon, A. Gali, and L. C. L. Hollenberg, Evidence for primal sp2 defects at the diamond surface: Candidates for electron trapping and noise sources, Adv. Mater. Interfaces 6, 1801449 (2019).

[43] S. Dhomkar, H. Jayakumar, P. R. Zangara, and C. A. Meriles, Charge dynamics in near-surface, variable-density ensembles of nitrogen-vacancy centers in diamond, Nano Lett. 18, 4046 (2018).

[44] D. Bluvstein, Z. Zhang, and A. C. B. Jayich, Identifying and Mitigating Charge Instabilities in Shallow Diamond Nitrogen-Vacancy Centers, Phys. Rev. Lett. 122, 076101 (2019).

[45] F. Dolde, H. Fedder, M. W. Doherty, T. Noebauer, F. Rempp, G. Balasubramanian, T. Wolf, F. Reinhard, L. C. L. Hollenberg, F. Jelezko, J. Wrachtrup, and T. Nöbauer, Electric-field sensing using single diamond spins, Nat. Phys. 7, 459 (2011).

[46] T. P. M. Alegre, C. Santori, G. Medeiros-Ribeiro, and R. G. Beausoleil, Polarization-selective excitation of nitrogen vacancy centers in diamond, Phys. Rev. B 76, 165205 (2007).

[47] H. Jayakumar, J. Henshaw, S. Dhomkar, D. Pagliero, A. Laraoui, N. B. Manson, R. Albu, M. W. Doherty, and C. A. Meriles, Optical patterning of trapped charge in nitrogen-doped diamond, Nat. Commun. 7, 12660 (2016).

[48] J. F. Barry, M. J. Turner, J. M. Schloss, D. R. Glenn, Y. Song, M. D. Lukin, H. Park, and R. L. Walsworth, Optical magnetic detection of single-neuron action potentials using quantum defects in diamond, PNAS 113, 14133 (2017).

[49] J.-P. Tetienne, R. W. de Gille, D. A. Broadway, T. Teraji, S. E. Lillie, J. M. McCoey, N. Dontschuk, L. T. Hall, A. Stacey, D. A. Simpson, and L. C. L. Hollenberg, Spin coherence of dense near-surface ensembles of nitrogenvacancy centres in diamond, Phys. Rev. B 97, 085402 (2018).

[50] J.-P. Tetienne, D. A. Broadway, S. E. Lillie, N. Dontschuk, T. Teraji, L. T. Hall, A. Stacey, D. A. Simpson, and L. C. L. Hollenberg, Proximity-induced artefacts in magnetic imaging with nitrogen-vacancy ensembles in diamond, Sensors 18, 1290 (2018).

[51] E. E. Kleinsasser, M. M. Stanfield, J. K. Banks, Z. Zhu, W. D. Li, V. M. Acosta, H. Watanabe, K. M. Itoh, and K. M. C. $\mathrm{Fu}$, High density nitrogen-vacancy sensing surface created via $\mathrm{He}^{+}$ion implantation of ${ }^{12} \mathrm{C}$ diamond, Appl. Phys. Lett. 108, 202401 (2016).

[52] S. Steinert, F. Dolde, P. Neumann, A. Aird, B. Naydenov, G. Balasubramanian, F. Jelezko, and J. Wrachtrup, High sensitivity magnetic imaging using an array of spins in diamond, Rev. Sci. Instrum. 81, 043705 (2010).

[53] M. Chipaux, A. Tallaire, J. Achard, S. Pezzagna, J. Meijer, V. Jacques, J. F. Roch, and T. Debuisschert, Magnetic imaging with an ensemble of nitrogen vacancy-centers in diamond, Eur. Phys. J. D 69, 166 (2015).

[54] B. J. Roth, N. G. Sepulveda, and J. P. Wikswo, Using a magnetometer to image a two-dimensional current distribution, J. Appl. Phys. 65, 361 (1989).

[55] A. Nowodzinski, M. Chipaux, L. Toraille, V. Jacques, J. F. Roch, and T. Debuisschert, Nitrogen-vacancy centers in diamond for current imaging at the redistributive layer level of integrated circuits, Microelectron. Reliab. 55, 1549 (2015). 